\title{
Condition assessment: From good choice of methods to reliable results that meet customer demand
}

\author{
Alexander Taffe* \\ HTW University of Applied Sciences, Department Building Analysis, Wilhelminenhofstr. 75A, D-12459 Berlin, Germany
}

\begin{abstract}
Condition assessment of structures reveals information of the inner structure and its condition. To be insightful the testing task has to be defined regarding the target state defined by the customer. Questions such as "What method should be applied?", "Where should be measured at what time?" and "How many measurements are sufficient?" have to be answered to guarantee reliable results. Often it is obvious that the additional use of non-destructive methods makes sense. But what method is the most appropriate and what strategy should be applied? This contribution will help to develop a strategy to achieve reliable results. Reliable results have to be accurate which means that reliable results have to be true and precise. According to the GUM (Guideline to the expression of Uncertainty in Measurement) the procedure how to evaluate data statistically will be demonstrated. Furthermore, it will be shown how statistically evaluated data can be used in static calculations to furnish the proof of the stability of the real as-built structure. This profoundly use of data contributes to make good and reliable decisions.
\end{abstract}

\section{Condition Assessment for maintenance of infrastructure}

Condition assessment reveals information of the inner structure, e.g. geometry, reinforcement ratio, number and depth of tendon ducts, as well as of its condition in terms of corrosion, compaction of the concrete or grouting of the ducts. But to be revealing appropriate methods have to be chosen and to be performed in a well-thought-out strategy. Table 1 demonstrates a list of the most useful methods (e.g. [1], [2]) divided in visual inspection, study of documents, invasive methods, non-destructive methods (NDT) and further methods using the gained knowledge.

Table 1 is not a complete list of appropriate methods but it presents the method (e.g. rebound hammer) in the context of the testing task (e.g. concrete compressive strength). Applying a certain method not always indicates the testing task. In case of potential mapping, it is not clear that the testing task is fulfilled just by localisation of corrosion active areas. It may also include delivering the plan for concrete removal. Thus, client and service provider should take time to define the testing task precisely to avoid misunderstandings.

It is also obvious that only the availability of a method will not guarantee solving the testing task - a strategy has to be developed. Fig. 2 gives four simple probably not complete - questions that could help to develop an effective strategy. The testing task can be very complex and will be defined by the material(s), structure, damage causes, exposition class, time of exposition etc.
Table 1. List of methods for condition assessment.

\begin{tabular}{|c|l|}
\hline Method & \multicolumn{1}{|c|}{ Example } \\
\hline Visual & $\begin{array}{l}\text { Visual Testing from simple or thorough } \\
\text { inspection or after impacts }\end{array}$ \\
\hline Study & $\begin{array}{l}\text { As-built drawings, protocols of regular } \\
\text { inspections, Building Logbook }\end{array}$ \\
\hline Invasive & $\begin{array}{l}\text { Carbonation depth, chloride profiles, core } \\
\text { drilling, sample taking etc. }\end{array}$ \\
\hline NDT & $\begin{array}{l}\text { rebound hammer ( concrete strength), magnetic } \\
\text { inductive methods (concrete cover), potential } \\
\text { mapping (detection of active corrosion), radar } \\
\text { and ultrasonic ( inner structure), magnetic stray } \\
\text { field (cracked prestressing steel) }\end{array}$ \\
\hline Further & $\begin{array}{l}\text { Static calculation (FEM, probabilistic), load } \\
\text { bearing test, monitoring, risk analysis }\end{array}$ \\
\hline
\end{tabular}

Starting in the upper left corner of Fig. 1 the measurand has to be defined. In case of compressive strength that could be core drilling or the application of the rebound hammer including the evaluation of the results. In case of rebound hammer the measurand is the $\mathrm{R}$ - or Q-value that is of no interest for the customer. His interest will be the class of the concrete compressive strength as a characteristic value. As well method combination - even invasive and NDT - might be beneficial. In case of pitting corrosion, the chloride content represented by a depth-profile in combination with concrete cover measurement using NDT might be

* Corresponding author: alexander.taffe $@$ htw-berlin.de 
very effective. Preliminary potential mapping could help to find the areas of most severe corrosion and to concentrate chloride depth-profiles in these areas. In case of cracked prestressing steel the measurand and the appropriate method might not be so easy to find. Magnetical stray field method (with the magnetic fluxdensity as measurand) might be the method of choice.

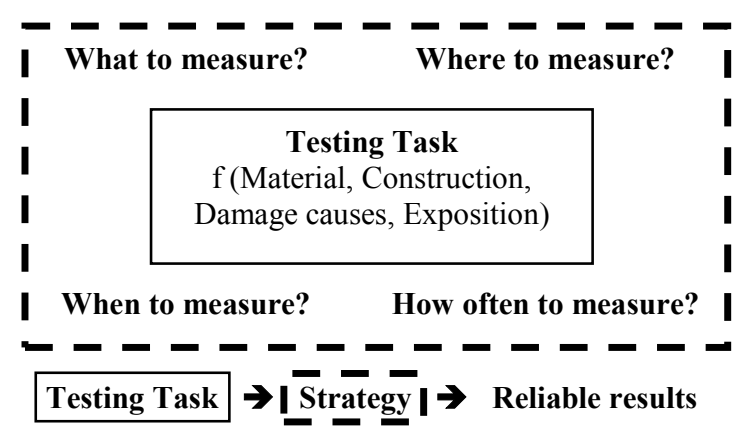

Fig. 1. Important questions to develop an effective strategy [3].

Once the measurand and the method have been identified according to the upper right corner of Fig. 1 the choice where the measurement will have to be carried out has to be selected. Respecting the technical needs to apply the method successfully, the strategy also includes an economic performance on-site. Not everything that is technically possible will help to solve the testing task. The good choice of specific areas that have to be measured done by an expert of the structure (e.g. personnel for the regular inspections) together with the NDT-expert will reduce the area, the measuring grid and the measuring time to a reasonable limit. This shows also the need for a multidisciplinary decision process.

The question "When to measure?" will be answered by the NDT expert. He knows about the boundary conditions under which a measurement will be successful. He will also know about the amount of fulfilled measurements. This includes the good choice of taking samples out of a basic population. These four questions might help to find a method that is applicable (1) from the physical point of view, (2) from the technical point of view concerning that particular construction (e.g. access, shape of the surface, reinforcement ratio, depth and size of objects etc.) and (3) under economic aspects (e.g. measuring time depending on grid and area) [3].

\section{NDT-CE methods for reliable results}

\subsection{NDT - Definition and overview}

As shown in Chap. 1 non-destructive testing methods (NDT) are part of the tool box used for condition assessment. In a first step it is helpful to understand what non-destructive means.

A definition for non-destructive testing is given in [4] (translated from German): „Technical process to quantify characteristic values of a material or product according to a certain procedure using the interaction of energy and material-property without affecting the serviceability. " Not affecting the serviceability is only one but not the main aspect. The main aspect is the interaction of energy. Thus, it is not surprisingly that energy is an appropriate parameter to classify NDTmethods. [5] gives the classification shown in Fig. 2.

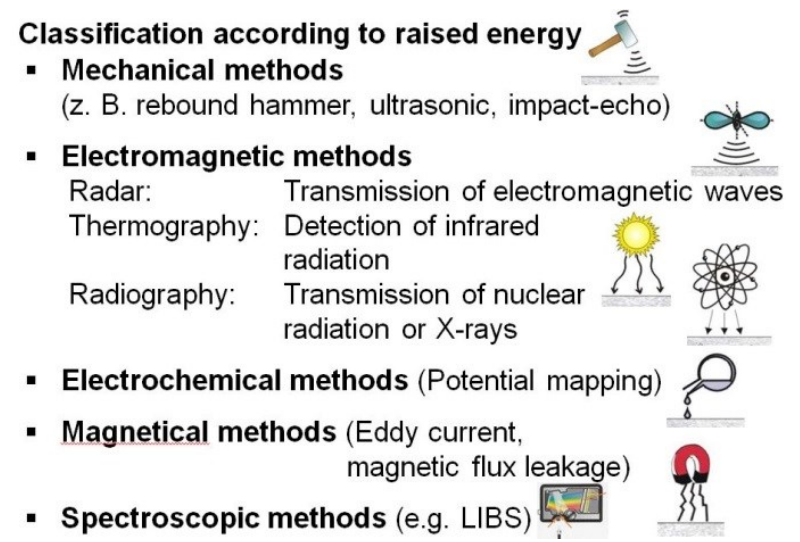

Fig. 2. Classification according to raised energy [5].

A third aspect of the definition mentioned above is that a material-property (e.g. R-value in case of rebound hammer as a parameter for hardness) has been measured, but only the characteristic values (e.g. concrete compressive strength) are of interest. The relation between measurand and result can be experimental (e.g. relation hardness and compressive strength) or based on a (sometimes simple) physical law (e.g. relation between measured transit-time and thickness depending on wavespeed). The NDT-expert has to understand these relations and has to evaluate the influence of certain boundary conditions in the result (e.g. roughness on the surface in case of ultrasonic-echo, concrete dampness in case of radar). Last but not least the NDT-expert has to evaluate the results in relation to the testing task. Therefore, it is not sufficient just to perform the measurement and to record the results. Non-destructive testing includes to furnish the proof that a certain limit has been exceeded, a limit of depth (e.g. in case of concrete cover) has been fulfilled or the detection of objects (of a certain size) is reliable. The idea of testing in NDT points out the importance of the clear identification of the testing task mentioned in Chap. 1.

\subsection{Strength and limitations of methods}

Within the last decades numerous of NDT-methods have been developed in civil-engineering (NDT-CE). Starting with the rebound hammer in the $1950 \mathrm{~s}$, concrete cover measurement, rebar detection and potential mapping became popular in the 1980s. These methods are often mentioned as classical NDT-CE methods.

At the end of the 1990s Radar and ultrasonic found more and more acceptance for reinforced (RC) structures. Both methods often declared as advanced NDT able to see the inner sight of RC structures. Imaging of results increased its capacity with the increasing performance of the hardware. The latest generation of devices uses the high computing capacity 
of tablets that are available at low budgets. But with the increasing application of NDT-CE methods questions arose about strength and the corresponding limitations of each method. Fig. 3 shows very schematically the strength of the most popular NDT-CE methods for the investigation of the inner structure. Each strength and limitation can also be proved physically, which not will be shown.

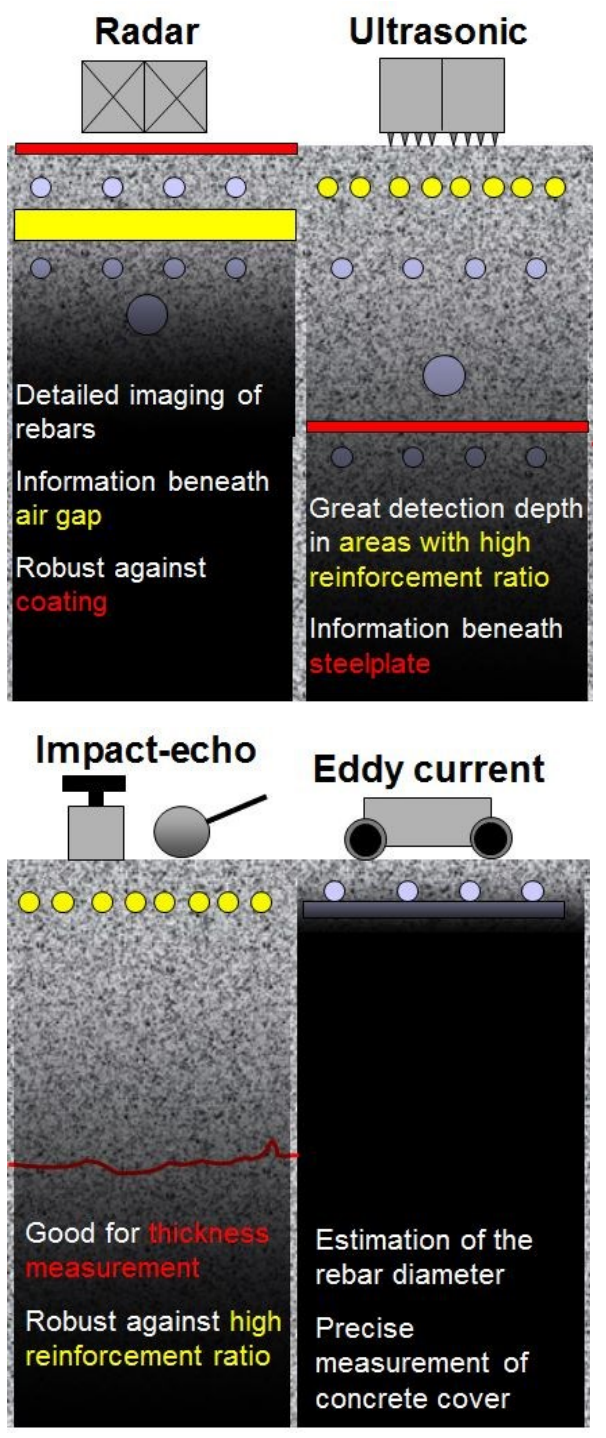

Fig. 3. Strength of NDT-CE methods according to [6].

While radar is good for the detection of reinforcement and tendon ducts due to the total reflection of metal objects, it is limited in case of rebars with a too close spacing. Ultrasonic is complementary; even areas with high reinforcement ratio or a metal plate can be penetrated. But ultrasonic energy is totally reflected in case of objects filled with air. This is beneficial in case of thickness measurement of a deboned screed or the detection of voids in a metal tendon duct. But ultrasonic is limited in multiple layer systems including air (e.g. thermal insulation). Radar will be perfect for these while ultrasonic cannot penetrate beneath an air-filled layer (e.g. sealing-layer).
Impact-echo is due to its much higher wavelength very powerful for the thickness measurement in highly reinforced structures such as inner tunnel shells but unsuitable for the imaging of the inner structure. Eddy current as a representative for the magnetic inductive methods measures the concrete cover very precisely $(+/-$ $1-2 \mathrm{~mm}$ ) but only up to approx. $6 \mathrm{~cm}$. Keeping these complementary strength and limitations in mind the appropriate method or method combination can be chosen very properly.

\subsection{POD to quantify limitations}

To predict if a testing task can be fulfilled requires more precisely information. First it has to be known up to what depth an object can be detected. POD (probability of detection) according to [7] and [8] has been established as a statistical tool to assess the reliability of a NDT system and helps to get an objective statement, if the existing NDT problem can be solved. This process is shown in Fig. 4 for the detection of a metallic reflector in concrete [9].
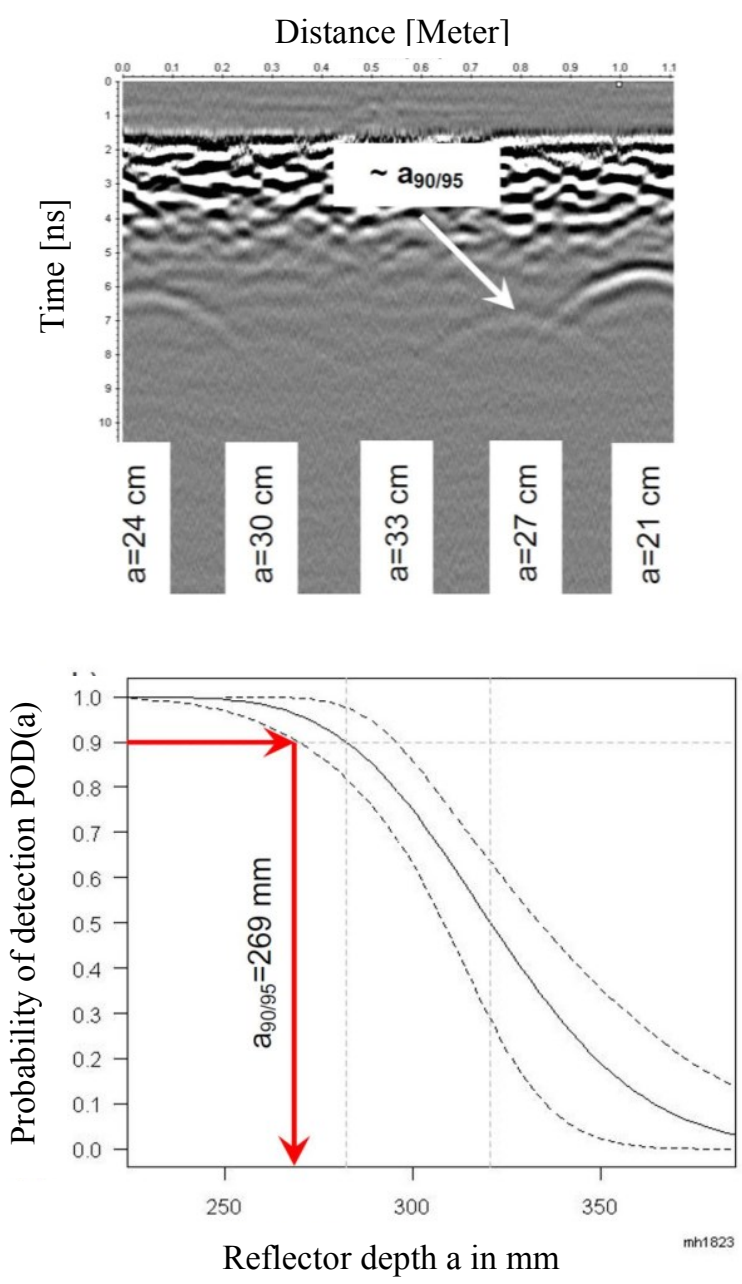

Fig. 4. Top: Radargram with rebars of $28 \mathrm{~mm}$ diameter of depth of 21 to $33 \mathrm{~cm}$. The hyperbola at $27 \mathrm{~cm}$ depth is the last one that is clearly visible. Bottom: POD-analysis with the a90/95-value at 269mm depth [9]. 
The top of Fig. 4 shows a radargram of a test specimen with five rebars of $28 \mathrm{~mm}$ diameter of depth from 21 to $33 \mathrm{~cm}$. The subjective data evaluation reveals that up to a depth of approx. $27 \mathrm{~cm}$ the hyperbola of the reflector can clearly be detected. This is in accordance with the result of the POD-analysis. The "a90/95" of $269 \mathrm{~mm}$ describes the depth, in which the reflector can be detected with radar in that particular case with a probability of $90 \%$ in 95 of 100 cases. This value states that up to that depth it is very unlikely to miss a rebar. Thus, this particular radar system can be successfully applied up to the depth of the a $_{90 / 95}$. This is a very objective procedure that allows even comparing one radar system with the other. Details are given in [10].

\subsection{Accuracy of results to guarantee their reliability}

If the applicability of a NDT-method has been proved, e.g. by POD (see Chap. 2.3), performance test or simple experience, the accuracy of the results has to be assured. According to the so-called target model from BAM [11] shown in Fig. 5 both components, trueness and precision, have to be fulfilled.

The trueness of the results, e.g. the input of the correct diameter in case of concrete cover measurement, has to be provided by well trained personnel. The precision of the results is expressed by quantifying the uncertainty of measurement (UoM). Only if the results are correct and in a reasonable way precise, they can be estimated "accurate".

The quantification of the UoM follows the procedure of the so-called GUM (Guide to the Expression of Uncertainty in Measurement, [12]), which has been internationally accepted. The basic idea is to quantify knowledge about the measurement process. It is represented by the identification of quantities influencing the results as shown in Fig. 6. These influence quantities (e.g. variance of the device, of the wave speed and unwanted changes of the thickness or unevenness) have to be quantified by statistical methods. They occur in the model equation giving the result of the measurement and in the formula for the combined total standard deviation. At the end of the process, there is a statistically evaluated result that allows drawing conclusions. Examples are given in [16]. Chap. 3 will rely on these results and their benefit for the use in static calculations.
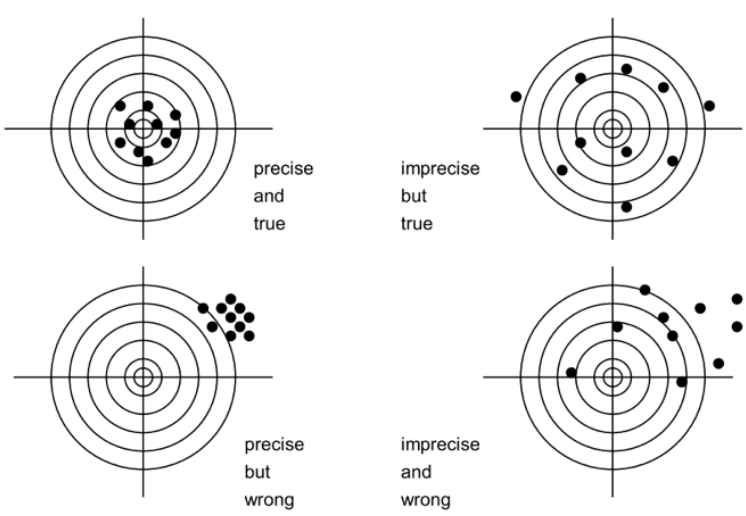

Fig. 5. Accuracy according to the target model from [11] depending on trueness and precision.

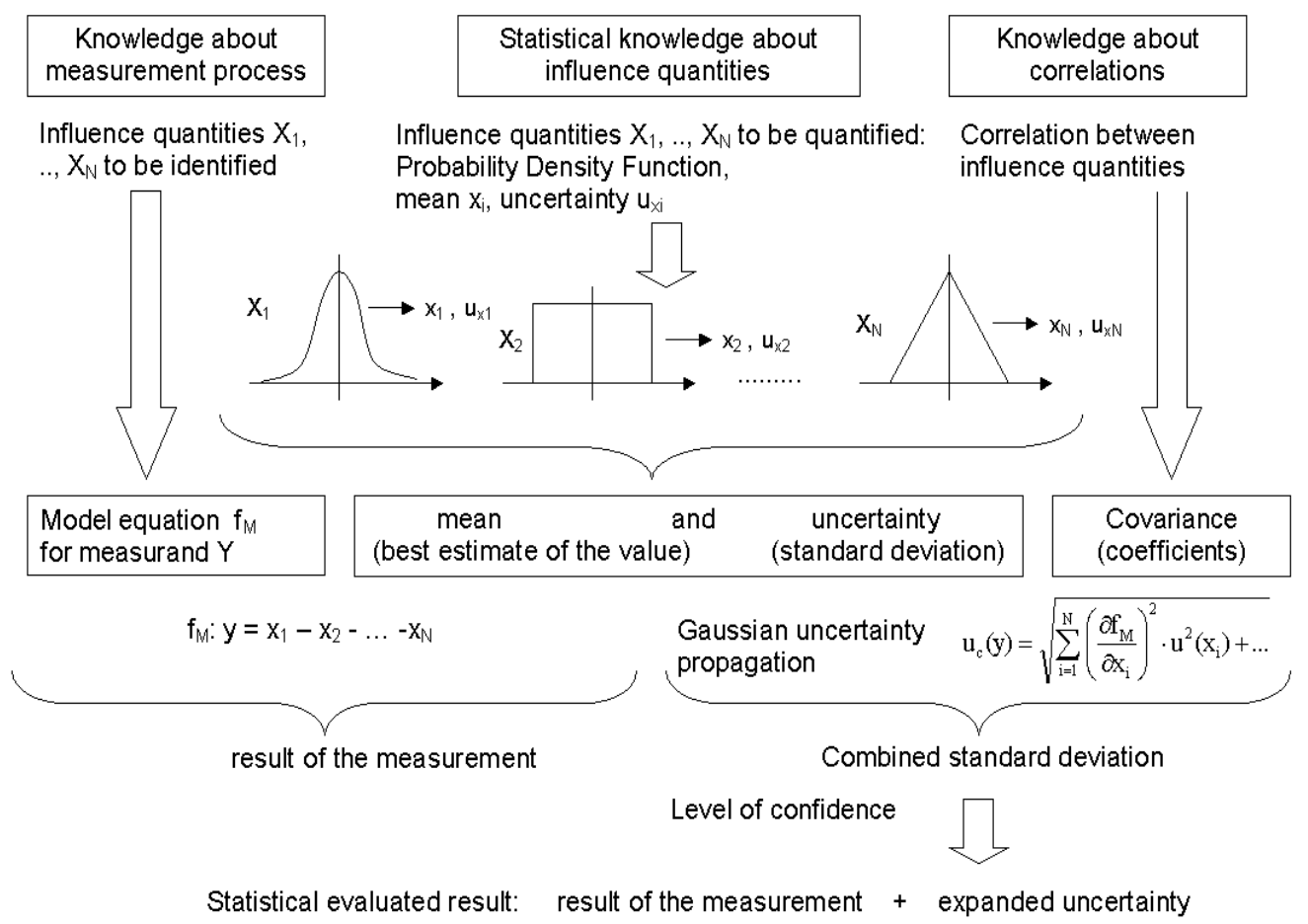

Fig. 6. Flowchart according to GUM [12] and based on Sommer [13]: Knowledge about the measurement process and quantities influencing the results will be quantified. A statistically evaluated result at the end of the process allows drawing reliable conclusions [14]. 


\subsection{Validation of NDT-methods}

Once the applicability has been proved (see Chap. 2.3) and the UoM has been quantified (see Chap. 2.4) under various conditions (e.g. variation of thickness, depth and size of objects, reinforcement ratio, concrete quality and quality of workmanship etc.) the validation of a particular testing task can be carried out. Following DIN EN ISO 17025 [15] validation is the confirmation by testing to furnish the proof that the requirements for a certain intended use can be fulfilled. As already published in [14] the customer - in some cases together with the service provider - expresses his requirements considering all necessary boundary conditions. If a testing method - suggested by the service provider succeeds to satisfy these requirements the validation process is completed. The validation process according to [5] consists of three steps as shown in Fig. 7:

- Characterisation of a testing method,

- Requirements of the client,

- Proof that the customer requirements have been fulfilled.

In Fig. 7 the importance of the UoM becomes obvious. In many cases it is the most important parameter to quantify the customer demand that has to be compared with results of characterisation of the method.

\section{Further use of reliable NDT-CE results in static calculations}

In civil engineering the use of limit state equations to assure the stability of RC structures has been established for decades. The semi-probabilistic approach is simple and very effective. But the recalculation of existing structures in case of increased load often fails. One reason is the high amount of safety that has been implemented in the partial factors for dead load (1.35) and traffic load (1.5). They include the variation of parameters (e.g. dimensions, concrete strength) that might occur due to the planning and construction process. But once the structure exists, the variation is much lower than estimated in the static calculation to construct a new building. For reasonable recalculation condition assessment as presented in the previous chapters allows evaluating the true as-built structure with its current variation of thickness, strength, position and track of reinforcement or tendon ducts. NDT-methods validated according to Chap. 2.5 may provide statistically evaluated results to be used in modified limit state equations.

The basic idea was already formulated in [10]. Using probabilistic analysis aims to calculate the probability of failure $\mathrm{p}_{\mathrm{f}}$ and the reliability index $\beta$ that are both mathematically linked. The basis of any probabilistic analysis is the limit state function. [16] that is presented at this conference gives an example for the internal bending moment. The ultimate limit state function represents the stochastic model as:

$$
\begin{gathered}
G=\Theta_{R, M} \cdot A_{s 1} \cdot f_{y} \cdot d \cdot\left(1-\frac{A_{s 1} \cdot f_{y}}{2 \cdot \chi \cdot b \cdot d \cdot \alpha \cdot f_{c}}\right) \\
-\Theta_{E, M} \cdot\left(M_{G}+M_{Q}\right)
\end{gathered}
$$

with

$d \quad$ effective depth of a cross-section (NDT-measurand),

$M_{G} ; M_{Q}$ - dead loads; traffic loads, $\Theta_{R} ; \Theta_{E}$ - uncertainty of resistance; of load effects. $\mathrm{A}, \mathrm{b}$ and $\mathrm{f}$ represent cross-sections, geometric properties and strength (stochastic input variables) and constants $(\alpha, \chi)$.

Except for the constants each variable can be quantified as a stochastic input variable by giving its distribution and their associated parameters (e.g. meanvalue and standard deviation in case of Normal distribution). This is mathematically done with specific software for probabilistic analysis, e.g. STRUREL. One option to quantify the precision of a measured parameter is to carry out the GUM-process described in Chap. 2.4. To measure a parameter more precisely, the width of the probabilistic density function (pdf) as it is shown in Fig. 8 from [10] will decrease. The gain of knowledge due to a more precise measurement is represented by $\sigma_{\mathrm{R} 1}<\sigma_{\mathrm{R} 2}$. It is obvious that this gain of knowledge will be paid back as an increase of reliability $\left(\beta_{\mathrm{R} 1}>\beta_{\mathrm{R} 2}\right)$ and low probability of failure. Gaining more knowledge about a structure may not always decrease $\sigma$ but will contribute to come to a more realistic result.

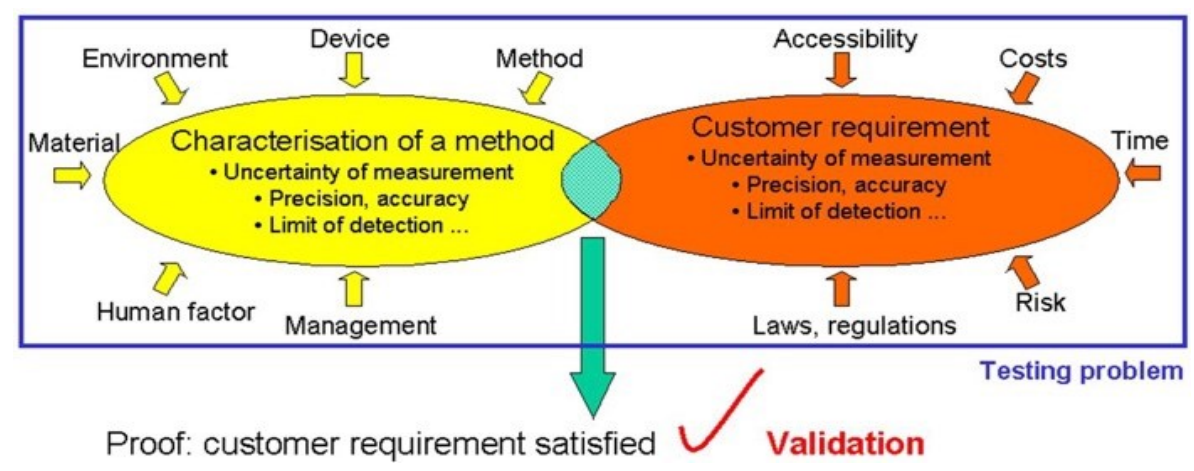

Fig. 7. Validation process according to [5] developed from [15] (taken from [14]). 


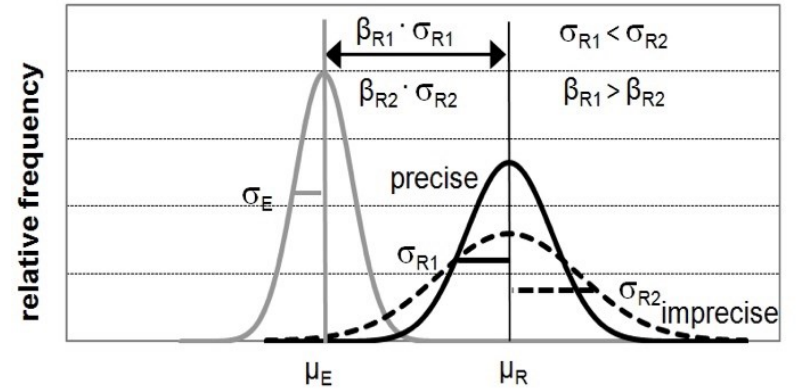

Values of $E$ and $R$

Fig. 8. Effect of precise and imprecise knowledge of the resistance R. Decreasing variance $\sigma$ in case of more precise knowledge will have an increasing effect on $\beta$, which means higher reliability [10].

In case of gaining more knowledge about the dead load it will help to decrease the partial safety index down from 1.35. In some cases that might help to prove sufficient stability by calculating the reliability index $\beta$ (see also [16]). The diagnostic tool of sensitivity analysis normally represented as a pie chart (see also [16]) helps to identify parameters that have the highest impact on the result of $\beta$. The measured parameter from NDT will be part of that chart; if its portion is relatively small it is the proof that the estimated UoM was sufficient and there is no need to spend more time and money in a more precise measurement.

\section{Summary and outlook}

Condition assessment is necessary - even in simple cases. At least the testing task has to be defined properly and a strategy has to be well thought out to meet the customer demand. Then the appropriate method - with NDT only as one part of the tool-box - will be identified by the service provider. The building owner may help to identify the "hotspot" where the measurement is mostly required. Well trained personnel will assure a correct data collection ("trueness"). If necessary the uncertainty of measurement ("precision") will be evaluated by the NDT-expert. Research institutions may contribute statistically evaluated data from validation. Well established guidelines show how to evaluate the uncertainty of measurement (GUM, [12]) and how to carry out a POD-analysis [8]. The use of NDT-results in static calculations - stated as NDT-SC - will contribute to a more realistic data base for the recalculation of $\mathrm{RC}$ structures. NDT-SC has a high potential to become an essential part of the structural engineering. Up to what depth the service provider will benefit from his data is up to him and depends on the complexity of the testing task. The main criterion of success at the end of the testing task is that the customer demand has been fulfilled.

\section{References}

1. DAfStb-Richtlinie: Schutz und Instandsetzung von Betonbauteilen, Part 1 to 4, Berlin (2001)
2. VDI6200: Structural safety of buildings, VDIRichtlinie (2010)

3. B. Hillemeier and A. Taffe: Aktuelle Regelwerke der Bauwerksdiagnostik, Bauphysik-Kalender (2012)

4. H.-U. Richter: Chronik der zerstörungsfreien Materialprüfung, DVS, Berlin (1999)

5. A. Taffe: Zur Validierung quantitativer zerstörungsfreier Prüfverfahren im Stahlbetonbau am Beispiel der Laufzeitmessung, DAfStb, Heft 574, Beuth Verlag Berlin (2008)

6. A. Taffe, S. Feistkorn and N. Diersch: Erzielbare Detektionstiefen metallischer Reflektoren mit dem Impulsradarverfahren an Beton In: Beton- und Stahlbetonbau 107 (2012) 7, pp. 442-450

7. A. P. Berens.: NDE Reliability Analysis, Reprinted from METALS HANDBOOK® Volume 17, 9th Edition: Non $\neg$ destructive Evaluation and Quality Control, University of Dayton Research Institute, ASM International (1989)

8. MIL-HDBK-1823A, Department of Defence Handbook, Nondestructive Evaluation System Reliability Assessment, 7. April 2009

9. S. Feistkorn: Gütebewertung qualitativer Prüfaufgaben in der zerstörungsfreien Prüfung im Bauwesen am Beispiel des Impulsradars. DAfStb, Heft 603, Beuth Verlag Berlin (2012)

10. T. Braml, A. Taffe, S. Feistkorn, O. Wurzer: Assessment of Existing Structures using Probabilistic Analysis Methods in Combination with Nondestructive Testing Methods. In: Structural Engineering International 23 (2013) 4, pp. 376-85.

11. W. Hässelbarth: Guide to the Evaluation of Measurement Uncertainty for Quantitative Test Results, EUROLAB Technical Report, Paris (2006)

12. JCGM 100:2008 Evaluation of measurement dataGuide to the expression of uncertainty in measurement

13. K.-D. Sommer, B. Siebert: Systematic approach to the modelling of measurements for uncertainty evaluation. In: Metrologia 43 (2006) 4, pp. 200-210

14. A. Taffe and Ch. Gehlen: Methodology for the validation of NDT-CE methods using transit time measurement, 7th International Symposium on Non Destructive Testing in Civil Engineering NDTCE 09, Nantes (2009)

15. DIN EN ISO/IEC 17025: General requirements for competence of test and calibration laboratories (2005-08)

16. S. Küttenbaum, A. Taffe, T. Braml and S. Maack : Reliability assessment of existing bridge constructions based on results of non-destructive testing, Proceedings of the International Conference on Concrete Repair, Rehabilitation and Retrofitting (ICCRRR), 19.-21.11.18, Cape Town, South Africa, in print 